\title{
FAST ILLUMINATED LOCK-IN THERMOGRAPHY: AN INLINE SHUNT DETECTION MEASUREMENT TOOL
}

\author{
S. Seren ${ }^{1}$, G. Hahn ${ }^{1,2}$, C. Demberger ${ }^{1,3}$, H. Nagel $^{4}$ \\ ${ }^{1}$ University of Konstanz, Department of Physics, P.O.Box X916, 78457 Konstanz, Germany \\ ${ }^{2}$ also with Fraunhofer Institute for Solar Energy Systems (ISE), Heidenhofstr. 2, 79110 Freiburg, Germany \\ ${ }^{3}$ now with Schmid GmbH + Co. Photovoltaik, Robert-Bosch-Str. 32, 72250 Freudenstadt, Germany \\ ${ }^{4}$ SCHOTT Solar GmbH, Carl-Zeiss-Str. 4, 63755 Alzenau, Germany
}

\begin{abstract}
This paper focuses on the fast spatially resolved detection of material and / or processing induced shunting using fast illuminated Lock-In Thermography (iLIT). With this technique shunting can be detected spatially resolved within a measurement time of only $1 \mathrm{~s}$ and thus the method in principle can be used for inline characterisation. Points addressed are the influence of Lock-In frequency on spatial resolution and the effect of a lowered illumination intensity for a better selectivity between shunting and recombination induced generation of heat. The method is tested for different common sources of shunting in standard industrial-type solar cells (points like shunts, cracks, poor edge isolation, contaminations) and all major types of shunts under investigation can be detected in times relevant for inline characterisation.
\end{abstract}

\section{INTRODUCTION}

Solar cells may suffer from poor parallel resistance causing losses in the efficiency potential due to shortcircuiting the emitter and base up to a certain degree. This is usually referred to as "shunting". Shunting causes a lowered fill factor (FF) and for significantly low shunt values even a drop in open circuit voltage $\left(\mathrm{V}_{\mathrm{oc}}\right)$. While FF and $V_{o c}$ are integral cell parameters, it is of interest to have a measurement tool available that can give locally resolved information about areas influenced by low shunt values.

Sources for low shunt values are material induced defects already present after crystallization (e.g. cracks, $\mathrm{SiN}_{\mathrm{x}}, \mathrm{SiC}$ or other precipitates) or processing related shunts (e.g. firing conditions, edge isolation).

\section{LOCK-IN THERMOGRAPHY}

Lock-In Thermography (LIT, [1]) detects spatially resolved heat sources in solar cells. The Lock-In technique enables an improvement of the thermal signalto-noise ratio which can be lowered from around $20 \mathrm{mK}$ for up to date infrared (IR) camera systems down to the $\mu \mathrm{K}$ range, dependant on the measurement time.

The modulated reference signal needed for the LockIn calculation is realized for the illuminated Lock-In Thermography by the solar cell itself under pulsed illumination via laser or LED panels. This method was invented simultaneously by the University of Konstanz (UKN) [2] and the FhG-ISE [3].

iLIT is a contactless measurement and provides a better signal compared to the conventional dark Lock-In Thermography (dLIT) [4], offering the possibility to use it as process monitoring tool even before metallisation. An application of iLIT therefore is an inline characterisation of heat dissipating areas (e.g. shunts) during solar cell processing.

\section{MEASUREMENT SETUP}

The thermography system used at UKN is based on a JADE IR camera and is now commercially available via the company InfraTec $\mathrm{GmbH}$ Dresden. The camera is sensible in the 3-5 $\mu \mathrm{m}$ range, the thermal noise $\sigma_{\mathrm{c}}$ is about $20 \mathrm{mK}$ and the image dimension is $320 \times 256$ pixels. The pulsed illumination is carried out via two LED panels at $880 \mathrm{~nm}$. Details can be found in [2].

\section{REDUCTION OF THE LOCK-IN FREQUENCY}

The Lock-In frequency used for a standard iLIT measurement with the setup described above is $15 \mathrm{~Hz}$. With 8 measurements (i.e. thermal images) within one Lock-In period a frame rate of the camera of $120 \mathrm{~Hz}$ results, which is the standard operating frequency of the used IR camera system.

A reduction of the Lock-In frequency increases the integrated thermal signal for a constant integration time of the camera's CCD sensor within one Lock-In period, but simultaneously decreases the spatial resolution as demonstrated in Figure 1 and Figure 2. 

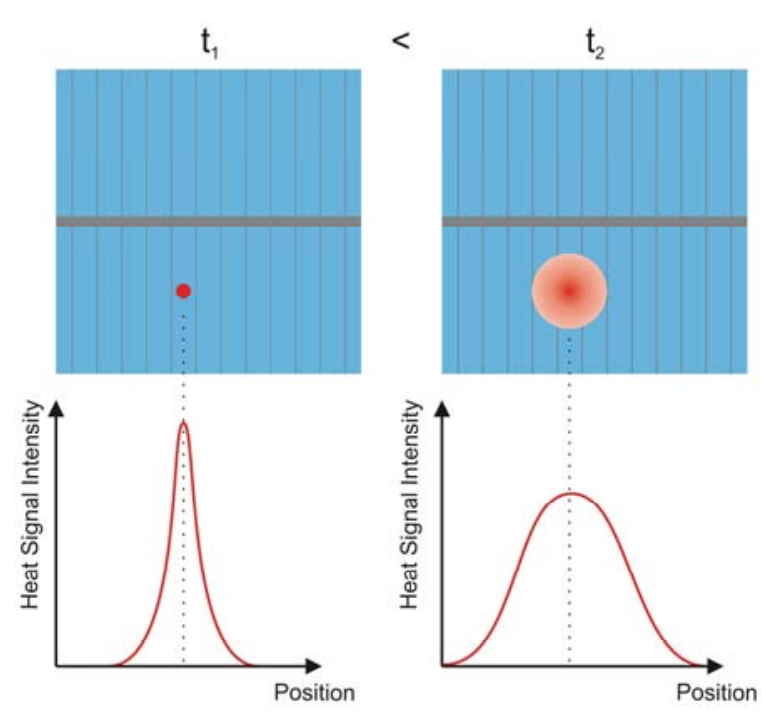

Figure 1: Thermal wave for two different times $t_{1}<t_{2}$ propagating from a shunt position in a solar cell.

In Figure 1 a thermal wave starts propagating in the solar cell from its point of generation (i.e. at a shunt position) isotropically in each direction. After a short time period $t_{1}$ the thermal wave propagated only a small spatial distance resulting in a strong but spatially localised heat signal. After a longer time period $t_{2}<t_{1}$ the thermal wave propagated in consequence a longer spatial distance resulting in a broadening of the thermal signal and thus in a reduced spatial localisation. However, the integrated areal heat signal has to be the same for both times $t_{1}$ and $t_{2}$ due to heat conservation. Thus, for the situation demonstrated in Figure 1 the integrated thermal signal within one single Lock-In period is higher for larger times $t_{2}>t_{1}$ due to the broadening of the propagating wave for $a$ constant Lock-In periodic time.

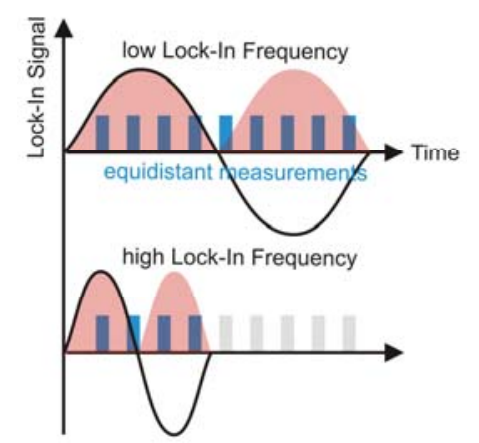

Figure 2: Contributions of discrete equidistant measurements performed by the camera's CCD sensor to the integrated thermal signal (red area) within a single Lock-In period for a low and a high Lock-In frequency.

A reduction of the Lock-In frequency and thus an enlargement of the measurement time interval causes the same effect, i.e. the integrated thermal signal increases (more contributions of the discrete equidistant measurements to the integrated thermal signal, upper part of Figure 2), but the spatial resolution decreases as demonstrated in the right part of Figure 1 due to a broadening of the area which contributes to the thermal signal.

\section{FAST ILIT MEASUREMENTS}

The influence of the measurement time on the noise level is demonstrated in Figure 3 where a frequency of $7.5 \mathrm{~Hz}$ was used for all measurements (illumination intensity 1 sun). Stronger shunts are already visible after a measurement time of $1 \mathrm{~s}$, but the signal-to-noise ratio is low and thus weaker shunts are hardly visible at this LockIn frequency.

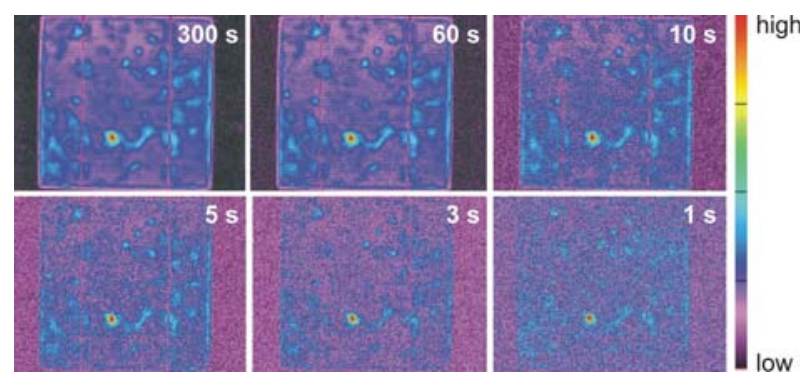

Figure 3: Conventional iLIT measurements of an industrial screen printed $12.5 \times 12.5 \mathrm{~cm}^{2} \mathrm{mc}$ solar cell for decreasing measurement times (Lock-In frequency $7.5 \mathrm{~Hz}$, illumination intensity 1 sun, $V_{\text {oc }}$ condition, same scaling for all measurements).

In order to enhance the thermal resolution for very short measurement times the Lock-In frequency was lowered to $1 \mathrm{~Hz}$, i.e. the measurement lasts only one single Lock-In period. Such fast iLIT measurements are shown in Figure 4 and demonstrate that strong point shunts are already visible within the very short measurement time of only $1 \mathrm{~s}$.

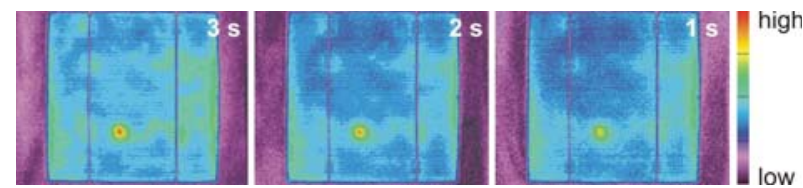

Figure 4: Fast iLIT measurements of the same solar cell as shown in Figure 3 but with a Lock-In frequency of $1 \mathrm{~Hz}$ (same measurement conditions as for Figure 3).

Besides point shunts originating e.g. from material induced defects such as precipitates penetrating the space charge region or over-firing of the front side metallisation, shunting of the cell edges is often observed due to an insufficient edge isolation during the cell process. Figure 5 shows an example for this kind of 
shunting which can be detected reliably with the fast iLIT measurement as well.
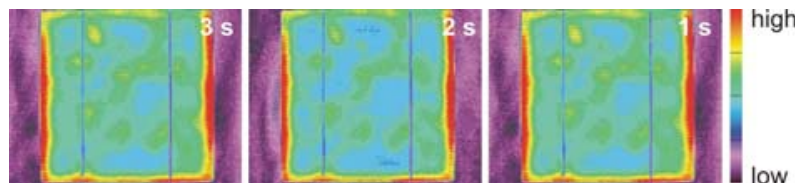

Figure 5: Fast iLIT measurements of an industrial screen printed $12.5 \times 12.5 \mathrm{~cm}^{2} \mathrm{mc}$ solar cell (same conditions as for the measurements shown in Figure 4).

As an example for a material and/or process induced shunt fast iLIT measurements of a $12.5 \times 12.5 \mathrm{~cm}^{2}$ EFG solar cell are presented in Figure 6 . A crack causing a strong shunt can clearly be identified at the lower right cell edge after a measurement time of $1 \mathrm{~s}$.
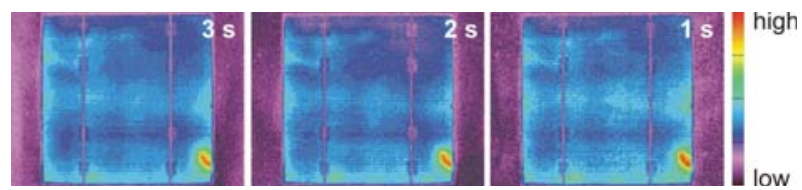

Figure 6: Fast iLIT measurements of an EFG solar cell (same conditions as for the measurements shown in Figure 4).

\section{ILLUMINATION INTENSITY}

The illumination intensity used for the Lock-In reference signal generation has an influence on the measurement. With lowering the illumination intensity and thus decreasing the excess carrier concentration the recombination current in the space charge region is reduced. As a result the influence of recombination active areas compared to the strength of the shunting signal of a particular shunt with a constant conductivity is less pronounced. The conductivity of the shunt does not directly depend on the illumination intensity and provides thus a more effective recombination path for excess charge carriers compared to the recombination in the space charge region.
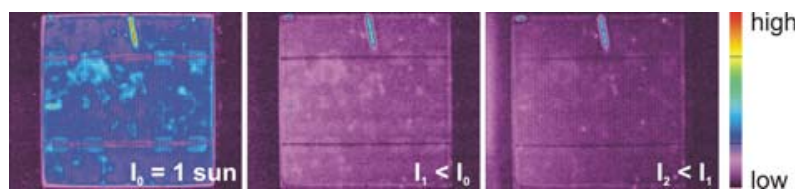

Figure 7: Conventional iLIT measurements of an industrial screen printed $12.5 \times 12.5 \mathrm{~cm}^{2} \mathrm{mc}$ solar cell for two reduced light intensities compared to 1 sun (Lock-In frequency $15 \mathrm{~Hz}$, measurement times $>400 \mathrm{~s}$, illumination intensity 1 sun (left) and two lowered light intensities (middle, right), $V_{o c}$ condition, same scaling for all measurements).
This effect is demonstrated in Figure 7: The recombination activity for two different lowered light intensities $I_{1}$ and $I_{2}$ compared to $I_{0}=1$ sun is noticeably reduced. Therefore, lowering the light intensity allows for detection of shunts more selectively.

It was found that lowering the intensity allows the detection of stronger shunts in very short measurement times even for a standard Lock-In frequency of $15 \mathrm{~Hz}$ (Figure 8, left).
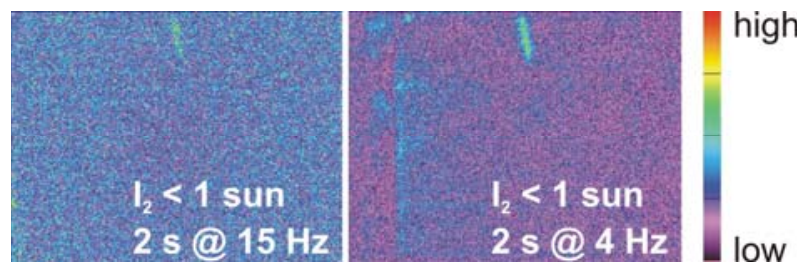

Figure 8: Fast iLIT measurements of the same solar cell as shown in Figure 7 but with a Lock-In frequencies of $15 \mathrm{~Hz}$ (left) and $4 \mathrm{~Hz}$ (right). Both measurements were carried out under the same reduced light intensity $\mathrm{I}_{2}<1$ sun as used for the measurement shown in Figure 7 (right) and $V_{o c}$ condition. Same scaling for both measurements.

\section{PROCESS MONITORING}

To check if e.g. Al contamination at the cell's front side can be detected with fast iLIT, a cell was deliberately contaminated with Al paste at the front side before firing. The cell has no front grid and no Al contact at the back.
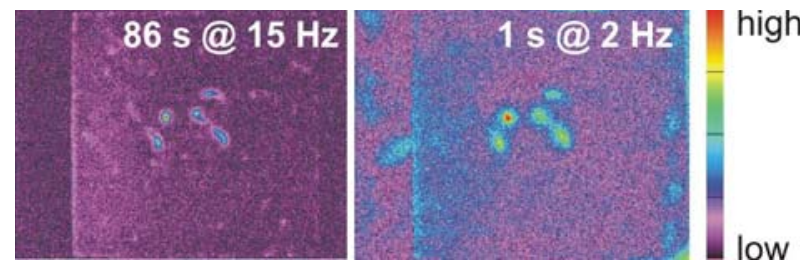

Figure 9: iLIT (left) and fast iLIT (right) measurements of a pn-structure (no front or back side metallisation) with an intentionally Al-contaminated front side after firing.

For a standard Lock-In frequency of $15 \mathrm{~Hz}$ and a lowered illumination intensity the detrimental effect of the $\mathrm{Al}$ contamination causing a shunt is clearly visible. Even for the $1 \mathrm{~s}$ fast iLIT measurement, the occurring shunts can clearly be detected (Figure 9, right).

\section{SUMMARY}

Fast Lock-In Thermography measurements have been carried out on industrial solar cells. The aim of this study was to demonstrate that most relevant types of harmful shunts can be detected reliably in very short measurement times down to $1 \mathrm{~s}$ relevant for inline processing control. 
The presented measurements show that besides point shunts, shunting caused by cracks as well as shunting at cell edges can be identified reliably within an inline relevant time frame.

The influence of an Al contamination at the front side could be detected within $1 \mathrm{~s}$ even without an Al back contact or a front grid present. This result proves that fast iLIT can be used for process monitoring inline even before metallization

\section{OUTLOOK}

It was demonstrated that with the fast iLIT technique measurement times of $1 \mathrm{~s}$ are enough to reliably detect different types of shunts. In combination with the latest generation of camera systems showing even better signalto-noise ratios compared with the one currently in use at UKN, inline detection of shunts should be possible even more easily and reliably.

\section{ACKNOWLEDGEMENTS}

Part of this work was funded by the EC in the CrystalClear (SES6-CT-2003-502583) project. The content of this publication is the responsibility of the authors.

\section{REFERENCES}

[1] O. Breitenstein, M. Langenkamp, Lock-In Thermography: Basics and Use for Functional Diagnostics of Electronic Components, Springer 2003

[2] M. Kaes, S. Seren, T. Pernau, G. Hahn, "Lightmodulated Lock-In Thermography for photosensitive pnStructures and Solar Cells", Progr. Photovolt.: Res. Appl. 12(5), 355, 2004

[3] J. Isenberg, W. Warta, "Spatially resolved evaluation of power losses in industrial solar cells by illuminated Lock-In thermography", Progr. Photovolt.: Res. Appl. 12(5), 339, 2004

[4] O. Breitenstein. J.P. Rakotoniaina, M. Kaes, S. Seren, T. Pernau, G. Hahn, W. Warta, J. Isenberg, "Lock-In thermography - a universal tool for local analysis of solar cells", Proc. $20^{\text {th }}$ EC PVSEC, 590-593, 2005

[5] R. O. Bell, J. P Kalejs, "Growth of silicon sheets for photovoltaic applications”, J. Mat. Res., 13, 2732-2739, 1998 